\title{
Spinal Myeloid Sarcoma in Two Non-Leukemic Patients
}

\author{
Songul Serefhanoglu, Hakan Goker, Salih Aksu, Yahya Buyukasik, Nilgun Sayinalp, \\ Ibrahim Celalettin Haznedaroglu and Osman Ilhami Ozcebe
}

\begin{abstract}
Myeloid sarcoma, formerly termed granulocytic sarcoma or chloroma, consists of neoplastic granulocytic precursors and myeloblasts. Isolated chloromas (granulocytic sarcomas) are rare tumors. Spinal complications of chloromas, such as cord compression secondary to epidural tumor or cauda equine syndrome have been described but are rare. We herein report two cases with spinal granulocytic sarcomas in non-leukemic patients. The case of a previously healthy 22-year-old man diagnosed with multiple spinal granulocytic sarcomas with no evidence of bone marrow or other hematological involvement is described. And, a 43-year-old woman diagnosed cervical spinal granulocytic sarcoma with no evidence of bone marrow or other hematological involvement is described. The tumor was totally removed by microsurgery. The histopathological examination was consistent with granulocytic sarcoma. Granulocytic sarcoma should be considered in the differential diagnosis of an epidural mass in patients with or without acute leukemia, because early diagnosis followed by appropriate combined chemotherapy and radiation may obviate surgical intervention and eventually prevent leukemic transformation.
\end{abstract}

Key words: myeloid sarcoma, spinal cord compression

(Intern Med 49: 2493-2497, 2010)

(DOI: 10.2169/internalmedicine.49.3878)

\section{Introduction}

Myeloid sarcoma (granulocytic sarcoma) has been defined as a tumor mass consisting of myeloid blasts with or without maturation occurring at an anatomical site other the bone marrow. Myeloid sarcomas are malignant neoplasms with the formation of invasive and destructive localized masses (1). Almost every site of the body can be involved; the skin, lymph node, gastrointestinal tract, bone, soft tissue and testis being more frequently affected. Two sites commonly involved with myeloid sarcoma are skin and lymph nodes $(2,3)$. Multiple anatomical sites constitute less than $10 \%$ of myeloid sarcoma cases. Myeloid sarcoma may occur de novo or as the initial manifestation of relapse in a patient with previously diagnosed acute myeloid leukemia without blood or bone marrow findings. Myeloid sarcoma develops in $2-8 \%$ of patients with acute myeloid leukemia $(4,5)$. Myeloid sarcomas involving the spinal cord and brain in patients without myeloid leukemia are rare. Spinal involvements are frequently misdiagnoses, usually as malignant lymphomas (2). In a review of the literature in 2002, Yamauchi and Yasuda found that 35 (47\%) of 74 patients initially were misdiagnosed (non-Hodgkin lymphoma, lymphosarcoma, histiocytic lymphoma, reticulum cell sarcoma, lymphocytic sarcoma, thymoma, myeloma, eosinophilic sarcoma and carcinoma). Eosinophilia of more than $300 / \mu \mathrm{L}$ was observed in 7/29 (24\%) of patients assessed for the blood leukocyte differentials and 22/31 (73\%) of patients had infiltration of eosinophilic infiltrate in the tumor. An inversion of chromosome 16 that may be associated with eosinophilia and eosinophil infiltration in granulocytic sarcoma tumor was not reported in these patients (6). Although eosinophilia and eosinophil infiltration in the tumor also occurs frequently in patients with granulocytic sarcoma, the inversion was not found in that review. Nonleukemic myeloid sarcoma patients may eventually develop acute leukemia $(2,7)$. Myeloid sarcomas of the spine and brain are usually located extradurally and associated with or lack bone involvement with or without bone destruction. The diagnosis of myeloid sarcoma of the spine and brain and its treatment has a very poor prognosis. We report two cases 
and review the literature.

\section{Case Report}

\section{Case 1}

A 22-year-old man presented with weight loss $(5 \mathrm{~kg}$ over two months), leukocytosis $\left(13,000 / \mathrm{mm}^{3}\right)$, eosinophilia $(40 \%)$ and a high sedimentation ratio $(48 \mathrm{~mm} / \mathrm{h})$. Bone marrow biopsy and aspiration test on right iliac bone were all negative for cytologic evidence of acute leukemia. Bone marrow was normocellular, with a significant increase in eosinophilic series $(30 \%)$ and marrow cytogenetic analysis revealed trisomy 8 . Thoracic and abdomen computed tomography was performed and there was no pathologic finding. One month later, he presented with a progressive backache and weakness in both legs causing difficulty to walk. At this time magnetic resonance imaging (MRI) of the spinal cord and brain was performed due to neurological deficits. Brain MRI showed that all skull bones were involved with myeloid sarcoma; tissue mass looked like lymphoma in supratentorial and extradural spaces. Full spinal MRI showed epidural thickened soft tissue in the cervical region which appeared as lymphoma, infiltrating the lymphoma mass and reaching all neural foramens and paravertebral spaces from the origin of the cervical spine (C2-3 levels). There was soft tissue mass in the epidural and paravertebral spaces between the cervical and thoracic spine (C7-T10 levels). The cord was invaded. Some soft tissue infiltrated the prevertebral and presacral spaces at the lumbar spine (L5 level) and sacral duct. Multiple biopsies were performed from the paravertebral mass between C5-T4 levels. Pathological examination of the tumor tissue showed positivity for myeloid markers: myeloperoxidase (MPO) and CD34, but negativity for $\mathrm{CD} 2$, CD3, CD5, CD20, CD30, CD56 and terminal deoxynucleotidyl transferase (Tdt). The final pathologic diagnosis was granulocytic sarcoma in the brain and spinal cord without bone marrow involvement (non-leukemic). The patient's blood cell count was normal: hemoglobin $12.9 \mathrm{mg} / \mathrm{dL}$, white blood cell count $6,500 / \mathrm{mm}^{3}$, platelet count $244,000 / \mathrm{mm}^{3}$ and $50 \%$ neutrophils, $26 \%$ eosinophils, $1 \%$ basophils, $19 \%$ lymphocytes, and $4 \%$ monocytes in peripheral smear. Complete biochemical panel was normal. Computed tomography (CT) of thorax and abdomen was normal except for only a vertebral metastatic focus. A bone marrow biopsy and aspirate (without eosinophilia), with flowcytometric analysis, showed normal findings. Cytogenetic study of the bone marrow cells revealed a normal karyotype (46,XY (20)). Molecular analyses were negative for each translocation and platelet-derived growth factor receptor (PDGFR). A lumbar puncture revealed acellular fluid with normal protein and glucose values. He immediately underwent cranial and spinal radiotherapy followed by systemic chemotherapy (Idarubicine plus cytarabine). However, the patient's condition worsened and he died due to septic shock.

\section{Case 2}

A previously healthy 43-year-old woman presented with left arm and leg pain that had worsened over a period of 3-4 months. No symptoms of paralysis were observed. She denied motor weakness and bowel or bladder incontinence. Magnetic resonance imaging showed an epidural $1.5 \mathrm{~cm}$ mass at the cervical spine (C6-7 level). Fluorodeoxyglucose (FDG) positron-emission tomography (PET) scan indicated that the lesion had a maximum standardized uptake value [SUV (max)] of 7. The patient underwent laminectomy of the cervical spine (C6-7 level) and the tumor was removed. The complete blood count and coagulation tests were normal, and no sign of leukemia was observed in the peripheral blood and bone marrow. Histological examination demonstrated fibroadipose connective tissue including diffuse cellular infiltrates. The great majority of the cells in the infiltrate consisted of myeloid precursors with large round to oval cells with slightly eosinophilic cytoplasm and round to oval vesicular nuclei, admixed with a minor population of more mature myeloid forms including eosinophils. Immunohistochemical stains showed tumor cells positive for myeloperoxidase (MPO) and negative for CD 3, 5, 7, 10, 20, 23 and Tdt. Mitotic figures were abundant. In consequence of this finding, the tumor was diagnosed as non-leukemic granulocytic sarcoma. Cytogenetic study of the bone marrow cells revealed a normal karyotype (46,XX (25)). Molecular analyses were negative for each translocation and PDGFRA. A lumbar puncture revealed cells infiltrated with myeloblasts. The patient underwent a course of external irradiation to the lumbar spine (total 50 Gy in 14 fractions). She was also given a full course of chemotherapy with mitoxantrone and a high dose of cytosine arabinoside according to the protocol of acute myeloid leukemia. She was receiving intrathecal chemotherapy and craniospinal irradiation. Two months later, MR imaging showed disappearance of the C6-7 mass and demonstrated a decreased maximum SUV of 3. However, the patient's condition worsened and she died due to ventricular fibrillation and cardiac arrest.

\section{Discussion}

Granulocytic sarcoma, also termed myeloid sarcoma or chloroma (King used 'chloroma' as the first term to describe this condition in $1853(10)$ ), is a rare malignant neoplasm resulting from the extramedullary proliferation of myeloid blasts with or without maturation or immature myeloid cells, which is reported frequently in acute myeloid leukemia, myelodysplastic syndromes with leukemic transformation, chronic myeloid leukemia with blastic crisis or in nonleukemic patients $(8,9)$. Granulocytic sarcoma is more than twice as common in children as in adults with acute myeloid leukemia and its overall incidence is 2.5$8 \%(5,11,12)$. Its prognosis is poor and most patients die within months. The WHO has renamed the condition as myeloid sarcoma and divided it into two major categories, 


\section{Spinal}

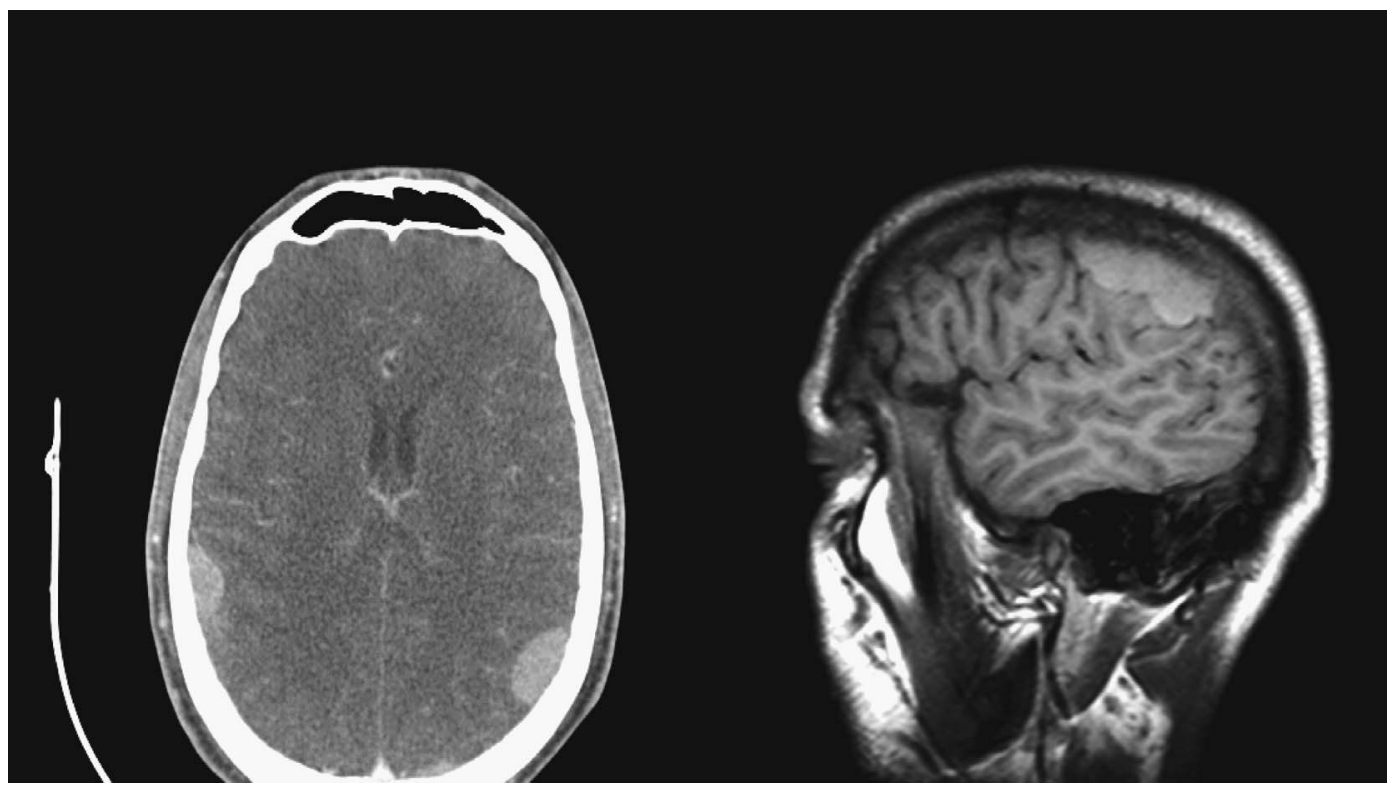

Figure 1. Granulocytic sarcomas in the brain (Case-1).

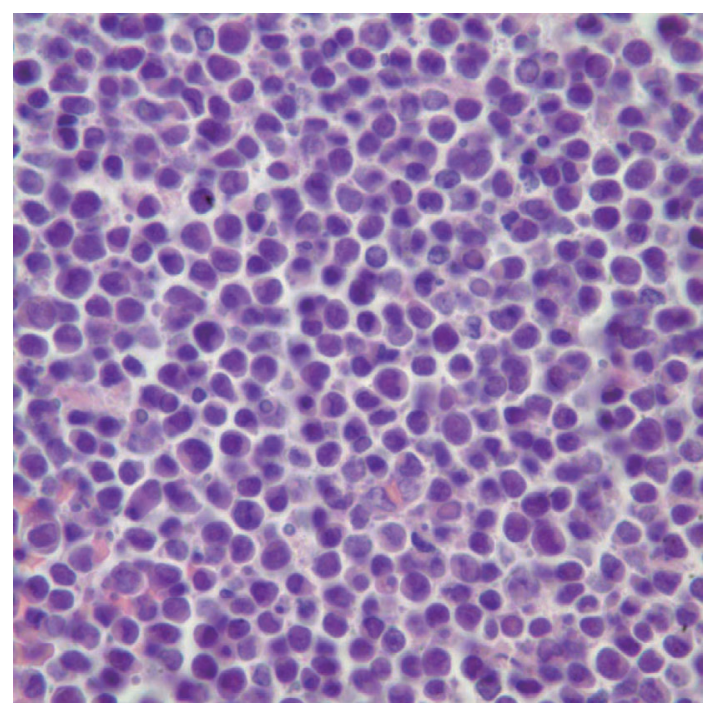

Figure 2. Hematoxylin and Eosin staining of myeloid sarcoma specimen (Case 1).

granulocytic sarcoma and monoblastic sarcoma (13).

Chloromas usually present as soft tissue or bony masses of the head and neck in patients with acute myeloblastic leukemia (AML) of the French-American-British M2 subtype. Granulocytic sarcoma rarely presents in the absence of systemic myeloid disease $(14,15)$. Moreover, $66-88 \%$ of patients with an isolated granulocytic sarcoma will develop AML at a mean of 9-11 months after diagnosis (16). Granulocytic sarcoma most commonly occurs in the soft tissues of the skin and bone, soft tissue of the head and neck (frequently the orbits), lymph node; rare cases involve the heart or small intestines and less often in the central nervous system and spinal cord (17-19). Involvement of the central nervous system (CNS) is rare, and spinal cord compression

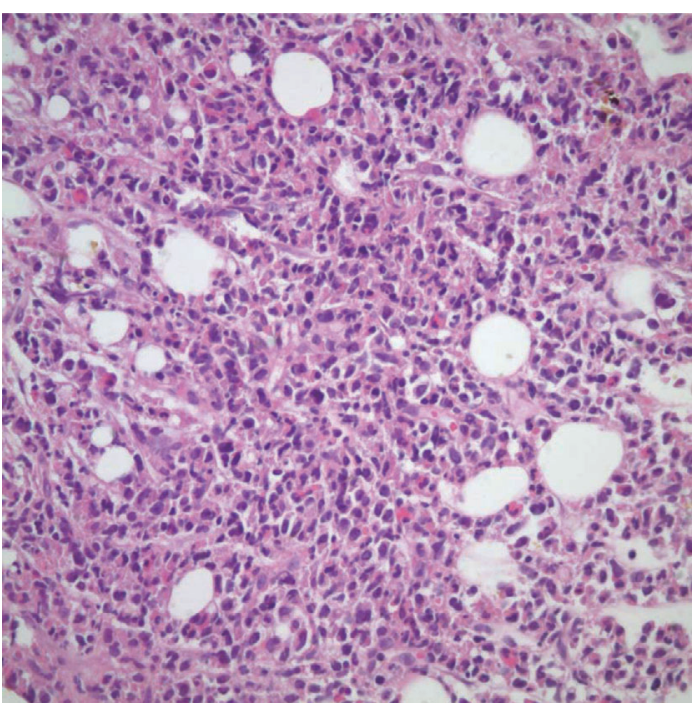

Figure 3. Hematoxylin and Eosin staining of myeloid sarcoma specimens (Case 2).

by granulocytic sarcoma is even more rare $(8,9)$.

Although involvement of every organ system has been reported in granulocytic sarcoma, the most common site is bone. The correct diagnosis of granulocytic sarcoma is obtained in only about $50 \%$ of non-leukemic patients due to its rareness and the histological and radiological similarities to malign lymphoma (20). Well-differentiated myeloid sarcomas are composed of eosinophilic myelocytes (21). The characteristic myeloid sarcoma phenotype is CD45, CD43, CD68 and myeloperoxidase positive and CD20 and CD45RO negative $(21,22)$. Myeloperoxidase, CD15 and neutrophil elastase are especially effective to differentiate granulocytic sarcoma from other tumors. Patients with nonleukemic granulocytic sarcoma may eventually develop 
acute leukemia $(2,7)$. Chromosome 8 abnormalities were the dominant abnormalities in the bone marrow and/or extramedullary sites. Translocation between chromosomes 8 and 21 is common in granulocytic sarcoma but nor requisite.

In MD Anderson Cancer Center data, granulocytic sarcoma occurred in $1.4 \%$ of patients with AML, and in $1.1 \%$ of patients with AML or high-risk myelodysplastic syndromes (MDS) (23). The median overall survival was 20 months (range, 1-75), median overall failure-free survival was 12 months (range, 1-75). The median survival of patients with chromosome 8 abnormalities was 12 months compared to 40 months in those without $(p=0.17)(23)$. In a literature review, the affected sites included cervical, thoracic, lumbar, sacral spinal level, bilateral renal enlargement, brain mass and cerebellum in non-leukemic patients. The thoracic spine was most commonly involved followed by the lumbar, sacral and cervical regions. The important clinical findings included pain (especially back pain), paresis, bowel or bladder incontinence, motor deficits, hypoesthesia, convulsions, cranial nerve palsies, papilloedema, cerebellar deficits, hemiparesis, aphasia, radicular pain, numbness and weakness of the extremities. Histological examination demonstrated neoplastic cells with small round and hyperchromatic nuclei proliferating in a diffuse pattern, and immature granulocytic cells and immunohistochemical staining demonstrated positive for myeloperoxidase (MPO), CD15, CD33, D56, CD34, CD45, LCA, chloroacetate esterase, monocytic antigen CD68, leukocyte common antigen CD45, neutrophil elastase and lysosome but negative for Tdt, CD20. The lesions were commonly isodense or hyperdense with surrounding edema on pre-contrast CT and they were markedly enhanced after contrast injection. The patients treated with surgical decompression, received additional AML type chemotherapy, radiotherapy, or bone marrow transplantation. Tsimberidou et al compared the clinical outcomes of matching granulocytic sarcoma patients with analogous AML patients in the MD Anderson Cancer Center ( $n=23$ granulocytic sarcomas, $n=1,720$ AML patients). Complete response rates were $69 \%$ in patients with granulocytic sarcomas and $57 \%$ in patients with AML. The 2-year eventfree survival (EFS) and overall survival (OS) rates were $32 \%$ and $18 \%(\mathrm{p}=0.08)$ and $43 \%$ and $29 \%(\mathrm{p}=0.11)$, respectively. They suggested that anti-AML therapy was highly effective in patients with nonleukemic granulocytic sarcomas and granulocytic sarcoma was associated with superior event-free survival and overall survival compared with acute myeloid leukemia (24-28).

Persisting eosinophilia occurs in hematologic malignancies such as idiopathic hypereosinophilic syndrome, chronic eosinophilic leukemia, systemic mast cell disease, chronic myeloid leukemia, chronic myeloproliferative disorders other than chronic myeloid leukemia and chronic myelomonocytic leukemia (29). Chromosomal abnormalities in eosinophiliaassociated hematological disease include chromosome bands 5q31-35 and 8p117, deletions of 20q, trisomy 8, and monosomy 7 (30). Chronic eosinophilic leukemia with trisomy 8 has been reported to be associated with transformation into acute leukemia and granulocytic sarcoma in the literature. Fujii et al reported a patient with hypereosinophilic syndrome, which, 8 years later, transformed into granulocytic sarcoma in the brain and, subsequently, into acute myelocytic leukemia. Trisomy 8 was demonstrated in cells from the bone marrow and cerebrospinal fluid (31). Guitard et al demonstrated a pediatric case of hypereosinophilic syndrome with trisomy 8 and terminal blastic transformation to mixed acute leukemia (32). WHO classification chronic eosinophilic leukemia is defined by an increase of $>2 \%$ blasts in the peripheral blood or of $>5-19 \%$ in bone marrow or evidence of clonality (33). In the present study, case 1 presented with eosinophilia with no blast in peripheral smear and bone marrow. Other potential causes of the eosinophilia were excluded.

Spinal complications of chloromas, such as cord compression secondary to epidural tumor or cauda equine syndrome have been described but are rare. The current two patients presented with spinal epidural tumors which caused spinal cord and cauda equine compression. Case 1, presented with eosinophilia and had multiple site myeloid sarcomas. Case 2, presented isolated cervical spinal granulocytic sarcoma. There was no leukemic involvement in bone marrow of two patients. The optimal therapy for patients with non-leukemic granulocytic sarcoma has not been determined. Our data indicated that AML-type intensive chemotherapy with or without radiotherapy was moderately effective. In conclusion, prompt diagnosis and adequate treatment are essential to achieve a good outcome.

\section{References}

1. Rappaport H. Tumors of the hematopoietic system, in Atlas of Tumor Pathology. In: Washington, DC, Armed Forces institute of Pathology, Section III, Fascicle 8. 1996: 241-243.

2. Meis JM, Butler JJ, Osborne BM, Manning JT. Granulocytic sarcoma in nonleukemic patients. Cancer 58: 2697-2709, 1986.

3. Traweek ST, Arber DA, Rappaport H, Brynes RK. Extramedullary myeloid cell tumors: An immunohistochemical and morphologic study of 28 cases. Am J Surg Pathol 17: 1011-1019, 1993.

4. Muss HB, Moloney WC. Chloroma and other myeloblastic tumors. Blood 42: 721-728, 1973.

5. Liu PI, Ishimaru T, McGregor DH, Okada H, Steer A. Autopsy study of granulocytic sarcoma (chloroma) in patients with myelogenous leukemia, Hiroshima-Nagasaki 1949-1969. Cancer 31: 948-955, 1973.

6. Yamauchi K, Yasuda M. Comparison in treatments of nonleukemic granulocytic sarcoma: Report of two cases and a review of 72 cases in the literature. Cancer 94: 1739-1746, 2002.

7. Byrd JC, Edebfield WJ, Dow NS, Aylesworth C, Dawson N. Extramedullary myeloid cell tumors in myelodysplastic-syndromes: not a true indication of impending acute myeloid leukemia. Leukemia Lymphoma 21: 153-159, 1996.

8. Balleari E, Panarello S, Capello E, et al. Granulocytic sarcoma: an unusual cause of spinal cord compression. Int J Clin Oncol 12: 234-237, 2007.

9. Neiman RS, Barcos M, Berard C, et al. Granulocytic sarcoma: a clinicopathologic study of 61 biopsy cases. Cancer 48: 1426-1437, 1981.

10. King A. Case of chloroma. Monthly J Med Sci (London) 7: 97, 
1853.

11. Muss HB, Maloney WC. Chloroma and other myeloblastic tumors. Blood 42: 721-728, 1973.

12. Pui MH, Fletcher BD, Langstone JW. Granulocytic sarcoma in childhood leukemia: imaging features. Radiology 190: 698-702, 1994.

13. Jaffe ES, Harris NL, Stein H, Vardiman JW. Pathology and genetics of tumours of haematopoietic and lymphoid tissues. WHO, IARC, 2001.

14. Landis MD, Aboulafia MD. Granulocytic sarcoma: an unusual complication of aleukemic myeloid leukemia causing spinal cord compression. A case report and literature review. Leuk Lymphoma 44: 1753-1760, 2003.

15. Scheinberg DA, Maslak P, Weiss M. Leukemias. In: Cancer: Principles and Practices of Oncology, chap. Devita VT, Hellman S, Rosenberg SA, Eds. Lippincott Williams and Wilkins, Philadelphia, 2001: 2427-2429.

16. Chevallier P, Mohty MB, Lioure B, et al. Allogeneic hematopoietic stemcell transplantation for myeloid sarcoma: a retrospective study from the SFGM-TC. J Clin Oncol 26: 1-4, 2008.

17. Kozelj M, Zorman D, Mrevlje B, Cernelc P, Zver S. Cardiac granulocytic sarcoma diagnosed by intracardiac echocardiographyguided biopsy. Int J Hematol 88: 101-103, 2008.

18. Kitagawa $Y$, Sameshima $Y$, Shiozaki $\mathrm{H}$, et al. Isolated granulocytic sarcoma of the small intestine successfully treated with chemotherapy and bone marrow transplantation. Int J Hematol 87: 410413, 2008.

19. Antic D, Vuckovic M, Elezovic I. Right atrial myeloid sarcoma causing superior vena cava syndrome. Br J Haematol 141: 134, 2008.

20. Williams MP, Olliff JF, Rowley MR. CT and MR findings in parameningeal leukemic masses. J Comput Assist Tomogr 14: 736742, 1990.

21. Roth MJ, Medeiros J, Elenitoba-Johnson K, Kuchnio M, Jaffe ES, Stetler-Stevenson M. (1995) Extramedullary myeloid cell tumors: An immunohistochemical study of 29 cases using routinely fixed and processed paraffin-embedded tissue sections. Arch Pathol Lab Med 119: 790-798, 1995.

22. Traweek ST, Arber DA, Rappaport H, Brynes RK. Extramedullary myeloid cell tumors: An immunohistochemical and morphologic study of 28 cases. Am J Surg Pathol 17: 1011-1019, 1993.

23. Tsimberidou AM, Kantarjian HM, Estey E, et al. Outcome in patients with nonleukemic granulocyctic sarcoma treated with chemotherapy with or without radiotherapy. Leukemia 17: 1100-1103, 2003.

24. Inoue $\mathrm{T}$, Takahashi $\mathrm{T}$, Shimizu H, et al. Spinal granulocytic sarcoma manifesting as radiculopathy in a nonleukemic patient- case report. Neurol Med Chir (Tokyo) 48: 131-136, 2008.

25. Park HJ, Jeong DH, Song HG, et al. Myeloid sarcoma of kidneys, the brain, and multiple bones in a nonleukemic child. Yonsei Med J 44: 740-743, 2003.

26. Mostafavi H, Lennarson PJ, Traynelis VC. Granulocytic sarcoma of the spine. Neurosurgery 46: 78-83, 2000.

27. Tsimberidou AM, Kantarjian HM, Wen S, et al. Myeloid sarcoma is associated with superior event-free survival and overall survival compared with acute myeloid leukemia. Cancer 113: 1370-1378, 2008.

28. Suzer T, Colakoglu N, Cirak B, Keskin A, Coskun E, Tahta K. Intracerebellar granulocytic sarcoma complicating acute myelogenous leukemia: a case report and review of the literature. J Clin Neurosci 11: 914-917, 2004.

29. Brito-Babapulle F. The eosinophilias, including the idiopathic hypereosinophilic syndrome. Br J Haematol 122: 203-223, 2003.

30. Bench AJ, Cross NCP, Huntly BJP, Nacheva EP, Green AR. Myeloproliferative disorders. Best Prac Res Clin Haematol 14: 531-551, 2001.

31. Fujii N, Ikeda K, Takahashi N, et al. Multilineage involvement in hypereosinophilic syndrome terminating in granulocytic sarcoma and leukaemic transformation with trisomy 8 . Br J Haematol 119: 716-719, 2002.

32. Guitard AM, Horschowski N, Mozziconacci MJ, et al. Hypereosinophilic syndrome in childhood: trisomy 8 and transformation to mixed acute leukaemia. Nouv Rev Fr Hematol 35: 555$559,1994$.

33. Jaffe E, Lee Harris NSHVJ. Tumours of Haematopoietic and Lymphoid Tissues. In: Chronic Eosinophilic Leukemia/Hypereosinophilic Syndrome. IARC Press, Lyon, 2001: 29-31.

(C) 2010 The Japanese Society of Internal Medicine http://www.naika.or.jp/imindex.html 